\title{
Diseño, evaluación y validación de un sistema de tratamiento de efluentes del proceso de curtido de pieles: caso Pieles del Sur E.I.R.L.
}

\section{(Design, evaluation and validation of an effluent treatment system for the leather tanning process: case Pieles del Sur E.I.R.L.)}

\author{
Víctor Muñoz Paz', Víctor Longa Cuayla², Alejandra Carreño Arispe³, José Aguilar Franco ${ }^{4}$
}

\begin{abstract}
Resumen
En el presente proyecto se diseñó un sistema de tratamiento para los efluentes del proceso de curtido de pieles; este sistema se evaluó a partir de la realización de ensayos de laboratorio para asegurar su viabilidad, y se validó el tratamiento mediante el análisis de efluentes provenientes de la planta ya instalada. El sistema tiene tres unidades: la primera es un pretratamiento que permite la oxidación de sulfuros para los baños de pelambre y calado, así como la precipitación de cromo para los baños de piquelado y curtido; la segunda es un tratamiento físico-químico para los efluentes generales del proceso que permite obtener efluentes tratados y sólidos decantados; la tercera es un tratamiento de lodos que permite obtener lodos acondicionados y de bajo volumen a ser recolectados para su disposición final. El análisis de los efluentes tratados demostró una reducción considerable en la concentración de químicos contaminantes que permite reutilizar el agua y acerca a la empresa al cumplimiento de los niveles máximos permisibles.
\end{abstract}

\section{Palabras clave}

Curtido de pieles; tratamiento de efluentes; contaminación.

\begin{abstract}
In this project a treatment system for the effluents of a leather tanning process was designed. This system was evaluated based on laboratory tests to ensure its viability, and the treatment was validated by analyzing effluents from the plant already installed. The system has three units: the first one is a Pretreatment that allows the oxidation of sulphides for the liming and draught baths, as well as the precipitation of chromium for the leather tanning baths; the second one is a Physical-Chemical Treatment for the general effluents of the process that allows to obtain treated effluents and decanted solids; the third is a sludge treatment that allows to obtain conditioned and low volume sludge to be collected for final disposal. The analysis of treated effluents showed a considerable reduction in the concentration of polluting chemicals that allows reusing the water and brings the company closer to compliance with the maximum levels allowed.
\end{abstract}

\section{Keywords}

Leather tanning; effluent treatment; environmental pollution.

\section{Introducción}

El curtido es el proceso de transformación de la piel de diferentes animales en cuero, para ello se añade un producto curtiente que sea capaz de penetrar en su estructura para bloquear los grupos aminos de la estructura coloidal; esto evita su degradación y aumenta el entrecruzamiento de las moléculas para obtener una estructura inerte a los efectos dispersantes del agua. Las etapas de producción requieren un alto consumo de agua, generando un alto nivel de efluentes líquidos que requieren de tratamiento (Artiga, 2005). 
La generación de este tipo de efluentes afecta directamente a la calidad del medio ambiente, razón por la cual el Gobierno de Perú ha generado una normativa que tiene por objetivo minimizar los efectos negativos de operaciones productivas como las que se dan en el proceso de curtido, entre otras: Decreto Supremo n. 019-97-ITINCI "Reglamento de Protección Ambiental para el Desarrollo de Actividades de la Industria Manufacturera", el Decreto Supremo n. 003-2002-PRODUCE "Límite Máximo Permisible de Efluentes para Alcantarillado de las Actividades de Cemento, Cerveza. Papel y Curtiembre", el Decreto Supremo n. 021-2009-VIVIENDA "Valores Máximos Admisibles de las Descargas de Aguas Residuales no Domésticas en el Sistema de Alcantarillado Sanitario".

La empresa peruana Pieles del Sur E.I.R.L. desarrolla las actividades de curtido de pieles, proceso productivo que antes del desarrollo del sistema de tratamiento propuesto demostró tener elevadas concentraciones de contaminantes y agentes químicos tóxicos en sus efluentes, tales como: cromo, sulfuro, SST (Sólidos en Suspensión Totales), DB05 (demanda bioquímica de oxígeno), DQO (demanda química de oxígeno) y nitrógeno amoniacal. Los resultados fueron obtenidos de diferentes monitoreos ambientales realizados a solicitud de la empresa para verificar el cumplimiento de las normas peruanas.

En general, los métodos tradicionales de producción de cueros en Perú incluyen metodologías que no aplican procesos de tratamiento óptimos para los contaminantes involucrados. Es así que, por ejemplo, estos métodos en promedio consumen entre el 60 \% y el $80 \%$ del cromo utilizado para el proceso de curtido, generando aguas residuales con altas concentraciones de cromo (Córdova, Vargas, Cesare, Flores y Visitación, 2014).

Los principales tipos de tratamiento para este tipo de aguas residuales se dividen principalmente en físico-químicos y biológicos. Los tratamientos físico-químicos incluyen operaciones como: desbaste, eliminación de sulfuros, homogeneización, decantación primaria, separación por flotación, coagulación-flotación, espesamiento, y secado de lodos; los tratamientos biológicos aplican procesos aeróbicos y anaeróbicos, e incluyen el tratamiento de lodos. Otra alternativa cuya utilización va en aumento es la utilización biorreactores de membrana (Artiga, 2005). Estos tratamientos tienen como objetivo la reducción de los niveles de contaminantes y agentes químicos tóxicos hasta niveles aceptables.

De acuerdo con la experiencia sobre oxidación y precipitación de compuestos sulfurosos descrita por Abia, Rejo y Vieira (2003), para el tratamiento de $1 \mathrm{~kg}$ de sulfuro se requiere de aproximadamente $4.9 \mathrm{~kg}$ de $\mathrm{KMnO} 4$ en un medio alcalino de $\mathrm{pH}$ 8; la adición de una solución polímero aniónico al $0.75 \%$ en peso permitirá recuperar $37.6 \mathrm{~kg}$ de residuos sólidos en $1 \mathrm{~m}^{3}$ de agua residual, obteniéndose una oxidación teórica de casi 100 \%. La desventaja de este proceso es el elevado uso de agentes químicos, generando gran cantidad de contaminantes sólidos. Es posible la sustitución del $\mathrm{KMnO} 4$ por $\mathrm{H} 2 \mathrm{O} 2$, siendo que para $1 \mathrm{~kg}$ de sulfuro se requiere de 12.9 l de esta solución al 30 \% en peso, vertiendo luego una solución de polímero aniónico al 1 \%, en peso, para separar los SST, obteniendo $1.1 \mathrm{~kg}$ de SST en $1 \mathrm{~m}^{3}$ de efluente resultante. La desventaja es el incremento de la temperatura durante la reacción (Abia, Rejo y Vieira, 2003).

Para lograr la oxidación de sulfuros es posible inyectar aire $\left(60 \mathrm{~m}^{3}\right.$ por $1 \mathrm{~m}^{3}$ de efluente aproximadamente), en un entorno de pH 13. La adición de $3.2 \mathrm{mg}$ de MnSO4 por $1 \mathrm{mg}$ de sulfato permitirá alcanzar una remoción promedio de 98.9 \% (Salas, 2005). Por otro lado, dado que los sulfuros presentan una baja solubilidad con iones metálicos, lograr su precipitación es posible en un medio de pH entre 8.5 y 8.0; se requerirá de $8.7 \mathrm{~kg}$ de Fe2(SO4) 3 por $1 \mathrm{~kg}$ de sulfuro, ade- 
más de una solución polímero aniónico al $2 \%$, en peso, para obtener 9.3 kg de SST por $1 \mathrm{~m} 3$ de efluente tratado. La precipitación de los sulfuros llega al 98 \% (Abia, Rejo y Vieira, 2003).

Por otro lado, según Moral (2010), la precipitación de cromo puede lograrse a partir de la utilización de $\mathrm{NAOH}$ como agente precipitante, incluyendo una etapa de floculación posterior. La utilización de $\mathrm{NaOH}$ para la precipitación de cromo es un método que requiere de aproximadamente $5 \mathrm{~g} / \mathrm{l}$, habiéndose realizado estudios que sugieren que la utilización de complejantes de cromo reduce la dosis a 0.5 - $3.5 \mathrm{mg} / \mathrm{L}$ (Córdova et al., 2014). Otra experiencia de precipitación de cromo sugiere la utilización de 3 tipos de agentes: $\mathrm{Na} 2 \mathrm{CO} 3, \mathrm{Ca}(\mathrm{OH}) 2$ y NaOH , alcanzando remociones de cerca del 99.7\% con dosificaciones de 2.3 - $3.3 \mathrm{~g}$ del segundo, y 2.6 a $3.5 \mathrm{~g}$ del tercero por $1 \mathrm{~g}$ de cromo presente; en este caso, la variación de ciertos parámetros en el experimento genera el incremento de hasta 4 veces las dosis para alcanzar el resultado descrito (Ortiz, 2013).

Respecto a las operaciones de coagulación, los coagulantes más utilizados en este tipo de efluentes son: $\mathrm{Ca}(\mathrm{OH}) 2$, Al2(SO4)3 y FeCl3. Según las pruebas realizadas por Islam, Misbahuzzaman, Kamruzzaman y Chakrabarty (2011), el segundo, y una mezcla de este con el tercero generan resultados altos de remoción de diferentes agentes contaminantes. En el caso de floculantes, Haydar y Anwar (2009) realizan un experimento de tratamiento de efluentes de curtiembre empleando floculantes aniónicos y catiónicos en concentraciones de $5 \mathrm{mg} / \mathrm{l}$, además de 100 mg/l de Al2(SO4)3 para los primeros, y 160 mg/l de este mismo compuesto para los segundos. En el caso de los efluentes de la industria del cuero, la utilización de floculantes en el tratamiento químico permite reducir casi el 50 \% de la utilización de coagulantes. Al utilizar un polímero catiónico se necesita de menos floculante, pero puede disminuir la cantidad de remoción de SST que se puede obtener debido a que en la reacción química estos dos entrar en un conflicto para llenar los espacios de los coloides del efluente (Haydar y Anwar, 2009).

El objetivo del presente trabajo fue diseñar y evaluar un sistema de tratamiento de efluentes para la empresa Pieles del Sur E.I.R.L., basado en los resultados obtenidos por experiencias previas (revisión bibliográfica), así como en los resultados de pruebas y ensayos de laboratorio realizados con los efluentes de la empresa para asegurar que el sistema permita la mejora de la calidad de estos. Además, una vez implementado, obtener y presentar resultados del análisis de efluentes tratados para demostrar la aplicabilidad de la propuesta. El trabajo se desarrolló en el marco del Concurso de Proyectos de Innovación de Empresas individuales - PITEl de Innóvate Perú - Ministerio de la Producción, a través del Contrato 493-PITEI-2016.

\section{Metodología}

\subsection{Material estudiado y parámetros analizados}

El material analizado en el presente estudio corresponde a muestras de los efluentes obtenidos del proceso productivo de curtido de pieles para determinar su calidad antes y después del tratamiento. Los ensayos de laboratorio para desarrollar el diseño y evaluación del sistema consistieron en el análisis del nivel de oxidación de sulfuros, precipitación de cromo y prueba de jarras para diferentes alternativas de pre-tratamiento y tratamiento físico-químico. Los parámetros analizados y las correspondientes normas aplicados en los ensayos tras la implementación del sistema propuesto se detallan en la tabla 1: 
Tabla 1. Parámetros analizados en los efluentes tratados

\begin{tabular}{|c|c|c|c|c|}
\hline Ensayo & Unidad & L.C.M (*) & Norma de Referencia & Título \\
\hline Aceites y grasas & $\mathrm{mg} / \mathrm{l}$ & 1.20 & $\begin{array}{l}\text { SMEWW-APHA-AWWA-WEF Part } \\
5520 \text { B, } 23 \text { rd Ed } 2017\end{array}$ & $\begin{array}{l}\text { Oil and Grease. Liquid-Liquid, } \\
\text { Partition-Gravimetric Method }\end{array}$ \\
\hline Cromo & $\mathrm{mg} / \mathrm{l}$ & 0.20 & $\begin{array}{l}\text { SMEWW-APHA-AWWA-WEF Part } \\
3030 \text { E. / Part } 3111 \text { B, } 23 \text { rd Ed. } \\
2017\end{array}$ & $\begin{array}{l}\text { Metals by Flame Atomic } \\
\text { Absorption Spectrometry. } \\
\text { Nitric Acid Digestion / Direct } \\
\text { Air-Acetylene Flame Method }\end{array}$ \\
\hline $\begin{array}{l}\text { Cromo } \\
\text { Hexavalente }\end{array}$ & $\mathrm{mg} / \mathrm{l}$ & 0.010 & $\begin{array}{l}\text { SMEWW-APHA-AWWA-WEF Part } \\
\text { 3500-CrB, } 23 \text { rd Ed. } 2017\end{array}$ & $\begin{array}{l}\text { Chromium. Colorimetric } \\
\text { Method }\end{array}$ \\
\hline $\begin{array}{l}\text { Demanda Bioquí- } \\
\text { mica de Oxígeno }\end{array}$ & $\mathrm{mg} / \mathrm{l}$ & 2.0 & $\begin{array}{l}\text { SMEWW-APHA-AWWA-WEF Part } \\
5210 \text { B, } 23 \text { rd Ed. } 2017\end{array}$ & $\begin{array}{l}\text { Biochemical Oxygen Demand } \\
\text { (BOD). 5-Day BOD Test }\end{array}$ \\
\hline $\begin{array}{l}\text { Demanda Quími- } \\
\text { ca de Oxígeno }\end{array}$ & $\mathrm{mg} / \mathrm{l}$ & 5 & $\begin{array}{l}\text { SMEWW-APHA-AWWA-WEF Part } \\
5220 \text { D, } 23 \text { rd Ed. } 2017\end{array}$ & $\begin{array}{l}\text { Chemical Oxygen Demand, } \\
\text { Closed Reflux, Colorimetric } \\
\text { Method }\end{array}$ \\
\hline $\begin{array}{l}\text { Nitrógeno } \\
\text { Amoniacal }\end{array}$ & $\mathrm{mg} \mathrm{N}-\mathrm{NH} 3 / \mathrm{l}$ & 0.10 & $\begin{array}{l}\text { SMEWW-APHA-AWWA-WEF Part } \\
\text { 4500-NH3 D, } 23 \text { rd Ed. } 2017\end{array}$ & $\begin{array}{l}\text { Nitrogen (Ammonia). Am- } \\
\text { monia-Selective Electrode } \\
\text { Method }\end{array}$ \\
\hline $\mathrm{pH}$ & Unidad de $\mathrm{pH}$ & 0.01 & $\begin{array}{l}\text { SMEWW-APHA-AWWA-WEF Part } \\
\text { 4500-H+ B, } 23 \text { rd Ed. } 2017\end{array}$ & $\begin{array}{l}\text { pH Value Electrometric } \\
\text { Method }\end{array}$ \\
\hline $\begin{array}{l}\text { Sólidos Suspendi- } \\
\text { dos Totales }\end{array}$ & $\mathrm{mg} / \mathrm{l}$ & 5 & $\begin{array}{l}\text { SMEWW-APHA-AWWA-WEF Part } \\
2540 \text { D, } 23 \text { rd Ed. } 2017\end{array}$ & $\begin{array}{l}\text { Solids. Total Suspended Solids } \\
\text { Dried at } 103-105^{\circ} \mathrm{C}\end{array}$ \\
\hline Sulfuro & $\mathrm{mg} / \mathrm{l}$ & 0.020 & $\begin{array}{l}\text { SMEWW-APHA-AWWA-WEF Part } \\
\text { 4500-S2- D, } 23 \text { rd Ed. } 2017\end{array}$ & $\begin{array}{l}\text { Sulfide. Methylene Blue } \\
\text { Method }\end{array}$ \\
\hline Temperatura & ${ }^{\circ} \mathrm{C}$ & 0.01 & $\begin{array}{l}\text { SMEWW-APHA-AWWA-WEF Part } \\
2550 \text { B. 23rd Ed. } 2017\end{array}$ & $\begin{array}{l}\text { Temperature. Laboratory and } \\
\text { Field Methods }\end{array}$ \\
\hline
\end{tabular}

Fuente: Informe de Ensayo n.: IE-19-3730, Analytical Laboratory E.I.R.L.

$\left.{ }^{(}\right)$Límite de cuantificación del método

\subsection{Sistema de tratamiento propuesto}

El sistema de tratamiento propuesto consta de cuatro procesos en tres unidades de tratamiento, las cuales permitirían la remoción de DBO y DQ0 en un 50 \% y $70 \%$, respectivamente; además de reducir las concentraciones de cromo y sulfuro a valores menores a $1 \mathrm{mg} / \mathrm{l}$ (figura 1).

A. Unidad de pretratamiento: consta de un sistema de oxidación de sulfuros para tratar los baños de pelambre y calado, y de un sistema de precipitación de cromo para el tratamiento de los baños de piquelado y curtido.

Los baños de pelambre y calado son descargados a un caudal promedio de $15 \mathrm{~m}^{3} / \mathrm{h}$ en una canaleta instalada al ras del piso debajo de los botales, direccionando el efluente al filtro de pelos para eliminar pelos y sólidos de gran tamaño. A continuación, el efluente es dirigido a una trampa de grasa con una capacidad de $3 \mathrm{~m}^{3}$, en el que permanece por aproximadamente 11.4 minutos para separar mediante flotación la grasa del efluente. Este último es entonces dirigido a un tanque de recirculación de baños de pelambre con una capacidad de $8 \mathrm{~m}^{3}$ a un caudal de $5 \mathrm{~m}^{3} /$ día; los efluentes permanecen en el tanque un máximo de 2 días para poder ser recirculados o con- 
tinuar con el proceso. Posteriormente, los efluentes pasan al tanque de oxidación de sulfuros que tiene una capacidad de $40 \mathrm{~m}^{3}$, en el que mediante la inyección de aire comprimido por una rampa de aireación se removerán los sulfuros por aproximadamente 8 horas.

Los baños de piquelado y curtido se descargan a un caudal promedio de $15 \mathrm{~m}^{3} / \mathrm{h}$ en una canaleta instalada al ras del piso debajo de los botales, direccionando el efluente a un primer filtro de bolsa que recolecta residuos gruesos y parte de las impurezas presentes. Después, el efluente se traslada a un tanque de precipitación de cromo con capacidad para $10 \mathrm{~m}^{3}$, en el que se sedimentan las aguas con cromo por aproximadamente 2 horas mediante decantación y la acción de un agitador de revolución baja. El efluente obtenido pasa por un segundo filtro de bolsa al mismo caudal que el primero, lo que permite separar el efluente de residuos sólidos con restos de cromo.

B. Unidad de tratamiento físico-químico: recibe los efluentes generales del proceso de curtiembre y los de la Unidad de pretratamiento.

Los baños generales se descargan a un caudal promedio de $15 \mathrm{~m}^{3} / \mathrm{h}$ en la canaleta instalada al ras del piso, debajo de los botales, siendo estos direccionados hacia una cámara de bombeo de $8 \mathrm{~m}^{3}$. El flujo de salida del efluente es el mismo gracias a la acción de una bomba sumergible que lo direcciona hacia un microfiltro de discos que remueve parte de los sólidos existentes. Luego, empleando una trampa de grasa de $6 \mathrm{~m}^{3}$, se separa la grasa a un caudal de $21 \mathrm{~m}^{3} / \mathrm{h}$ por los siguientes $17 \mathrm{mi}$ nutos aproximadamente. Estos efluentes, junto con los provenientes del tanque de oxidación de sulfuros, son contenidos en el tanque de homogeneización de 1000 $\mathrm{m}^{3}$, el cual trabaja a un caudal promedio de $10 \mathrm{~m}^{3} / \mathrm{h}$ para, mediante inyección de aire por una rampa de aireación, separar los SST y parte del Nitrógeno Amoniacal por las siguientes 10 horas. Una vez aireados y homogeneizados, los efluentes son enviados al mixer de coagulación y floculación, agitadores estáticos de espiral colocados en las tuberías para mezclar el coagulante y floculante a un caudal no menor de $1 \mathrm{~m}^{3} / \mathrm{min}$. El efluente resultante es desaguado en el decantador primario 0 sedimentador de $20 \mathrm{~m}^{3}$, en donde permanece aproximadamente por 1.3 horas a un flujo continuo de $10 \mathrm{~m}^{3} / \mathrm{h}$ para remover el DBO5 y DQO contenidos en los flóculos formados, obteniendo el efluente tratado y sólidos decantados que son enviando al tratamiento de lodos.

C. Unidad de tratamiento de lodos: recibe los sólidos decantados del tratamiento físico-químico.

Los lodos resultantes son recibidos en un tanque de almacenamiento de lodos de 2 $\mathrm{m}^{3}$ a un caudal promedio de $5 \mathrm{~m}^{3} / \mathrm{h}$, donde permanecen por aproximadamente $24 \mathrm{mi}$ nutos. A través de una válvula de lodos y una bomba de lodos se trasladan los lodos a un tanque floculante para ser mezclados con sales y polímeros, obteniendo un lodo adensado de fácil extracción. Este último, que tiene entre $2 \%$ y 3 \% de contenido de sólidos, pasa por un sistema mecanizado de deshidratación de lodos, obteniendo un lodo acondicionado y de bajo volumen, listo para su disposición final. 
Figura 1. Esquema del sistema de tratamiento de efluentes

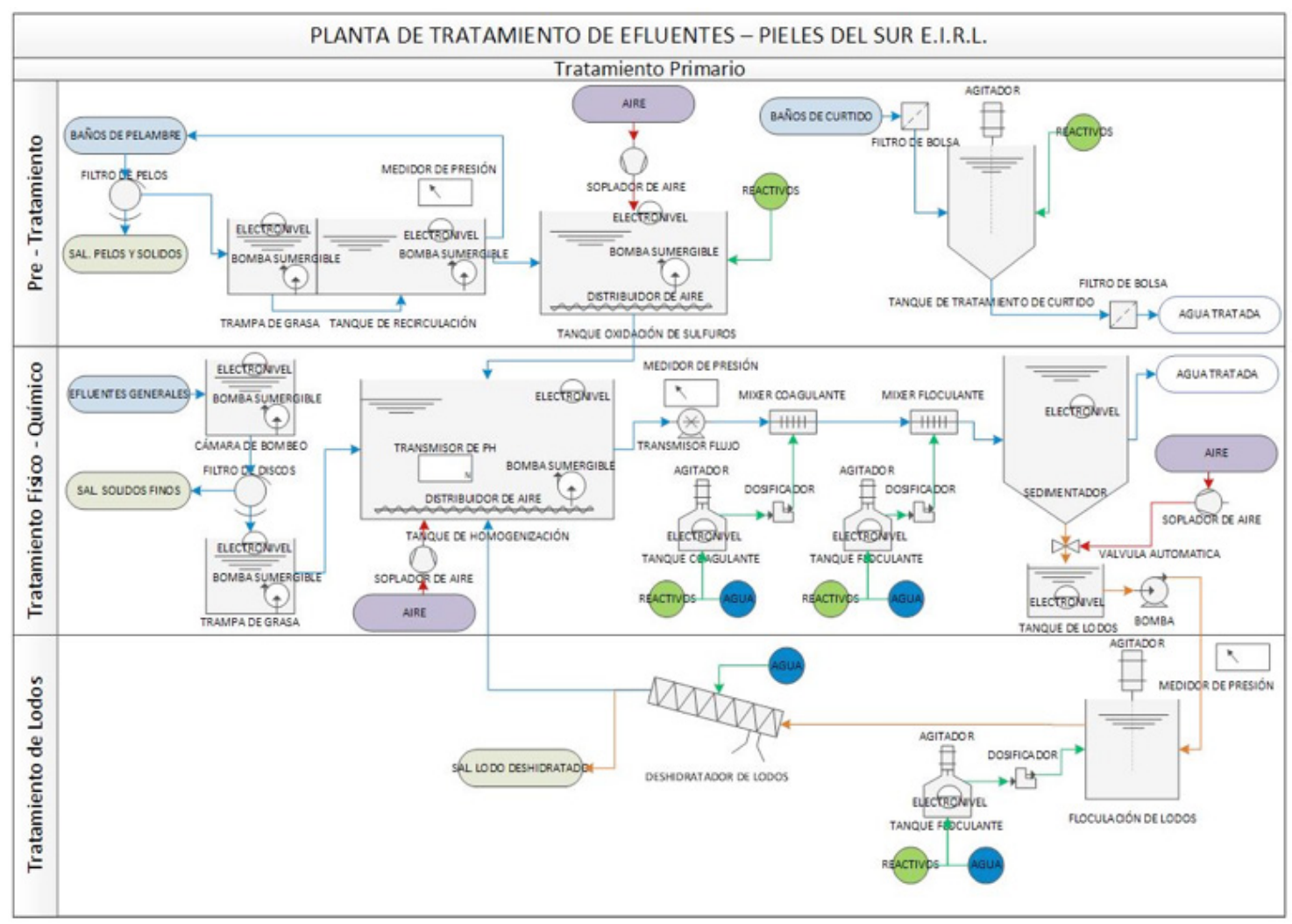

Fuente: Proyecto Convenio n. 493-Innóvate Perú PITEI.

El diseño del sistema de tratamiento de efluentes propuesto fue evaluado a partir de ensayos de laboratorio para garantizar la reproductibilidad del modelo antes de su implementación. Se realizó:

- Pretratamiento de efluentes de pelambre: análisis de una muestra de los efluentes de pelambre para conocer el máximo contenido de sulfuros, así como un ensayo de oxidación de sulfuros en este tipo de efluentes. El ensayo, que se realizó en un depósito de 4 I de capacidad, implicó la inyección de aire, empleando como referencia la oxidación máxima posible mediante $\mathrm{H} 2 \mathrm{O} 2$ y MnSO4.

- Pretratamiento de efluentes de curtido: utilización de agentes basificantes de $\mathrm{NaOH}$ (las dosis se determinaron mediante ensayos de 4 tipos de tratamiento) para lograr la insolubilización del cromo, logrando su precipitación en forma de una lechada densa de color celeste, dejando un sobrenadante transparente que evidencia la no presencia de sales de cromo.

- Tratamiento físico químico de efluentes generales: realización de pruebas de jarras para determinar si los procesos de coagulación / floculación son viables para tratar los efluentes de la empresa. Las pruebas se realizaron con 6 agentes, 2 agentes coagulantes que son sales cargadas y 4 floculantes que son polímeros orgánicos e inorgánicos 
con carga positiva, negativa y neutra. Las pruebas realizadas se hicieron para determinar la mejor combinación de agentes químicos que permita la separación del efluente en sobrenadante y flóculos sedimentados, y posteriormente determinar qué combinación dará la mejor separación de fases en las dosis; para esto último se preparó un diseño experimental factorial que involucro a las distintas concentraciones de los insumos.

\section{Resultados y discusión}

\subsection{Resultados de los ensayos de laboratorio}

Los ensayos de laboratorio permitieron garantizar la reproductibilidad del modelo diseñado antes de su implementación:

- El ensayo de oxidación de sulfuros permitió crear una curva de oxidación presentada en la figura 2, en la cual se observa el tiempo necesario para la reducción de la concentración de sulfuros de 490 mg/l a 10 mg/l. El caudal de entrada para esta oxidación fue de $1.700 \mathrm{ml} / \mathrm{min}$, lo cual indica que el requerimiento de oxígeno es alto; es importante mencionar que el tiempo de contacto de las microburbujas de aire en la unidad experimental es mínimo, mientras que en el caso de la poza donde se llevará a cabo la oxidación el tiempo de contacto será mayor, disminuyendo considerablemente los requerimientos de aire a ser inyectado. Por otro lado, en la figura 3 se puede evidenciar el incremento de oxígeno disuelto a medida que a medida que se reduce el contenido de sulfuros, siendo el nivel máximo alcanzado de $41.7 \%$, lo cual implica la mínima presencia de estos compuestos en los efluentes ya oxidados.

Figura 2. Curva de oxidación de sulfuros

\section{Oxidación de Sulfuros}

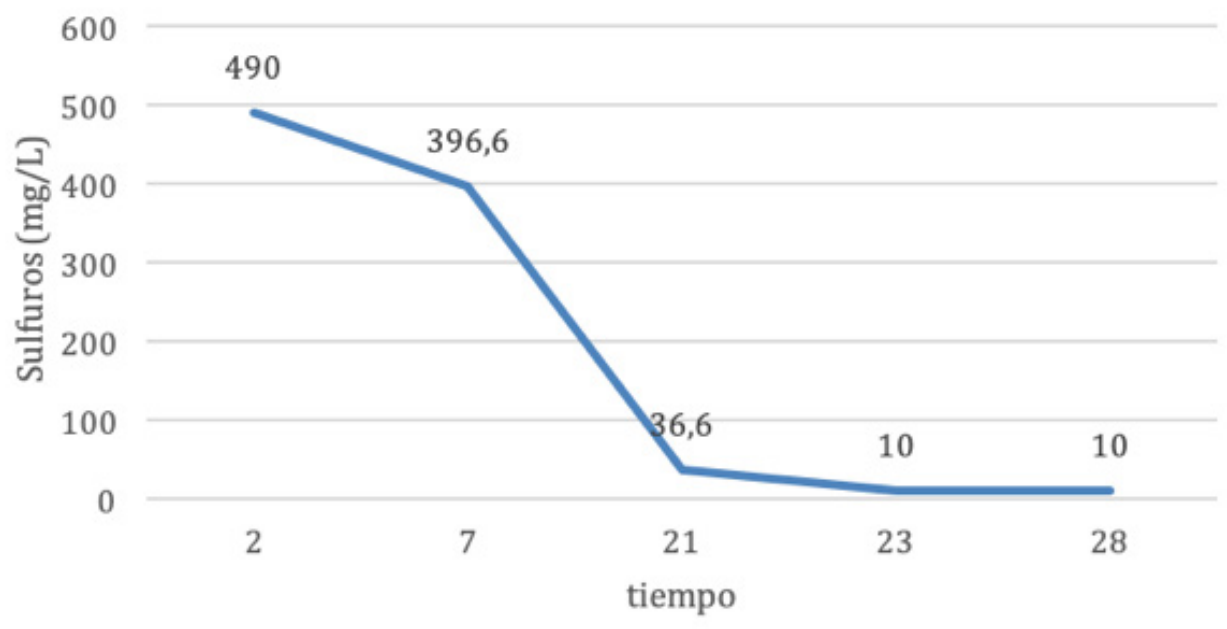

Fuente: Ensayos de pretratamiento y tratamiento de efluentes en la empresa Pieles del Sur-Arequipa. 
Figura 3. Porcentaje de requerimiento de oxígeno disuelto en función al tiempo

\section{Retención de \%OD}

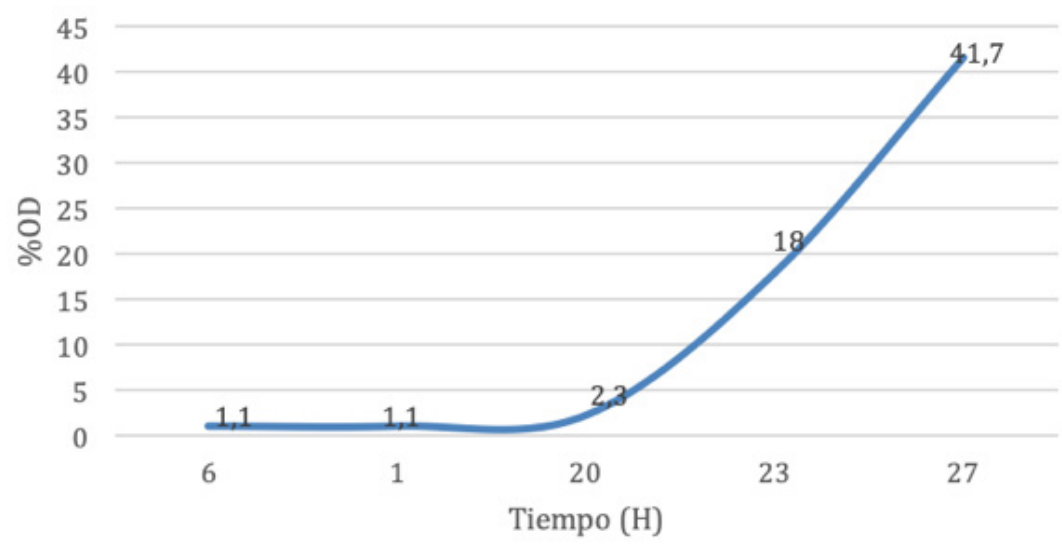

Fuente: Ensayos de Pre-tratamiento y tratamiento de efluentes en la empresa Pieles del Sur-Arequipa.

- El ensayo de precipitación de cromo evidenció durante la utilización de agentes basificantes que el mayor nivel de remoción de cromo se alcanzó al llegar a un pH de 9.5. Los 4 tipos de tratamiento permitieron una reducción de la turbidez de los efluentes de 871 NTU a 325NTU en el tratamiento $1(12 \mathrm{~g} / \mathrm{l})$, 1 NTU en el tratamiento $2(6 \mathrm{~g} / \mathrm{l}), 126 \mathrm{NTU}$ en el tratamiento $3(3 \mathrm{~g} / \mathrm{l})$, y 173 NTU en el tratamiento $4(1.5 \mathrm{~g} / \mathrm{l})$; se denominó tratamiento 5 a un ensayo sin basificantes (sin variación de turbidez). La figura 4 muestra el porcentaje de sedimentación lechada de cromo para los tratamientos 1, 3 y 4 (curva roja), tratamiento 2 (curva azul), y tratamiento 5 (curva amarilla); de acuerdo con estos resultados, a las 14 horas se alcanza una sedimentación máxima de casi 100 \% en ambos casos.

Figura 4. Sedimentación de la lechada de cromo con respecto al tiempo

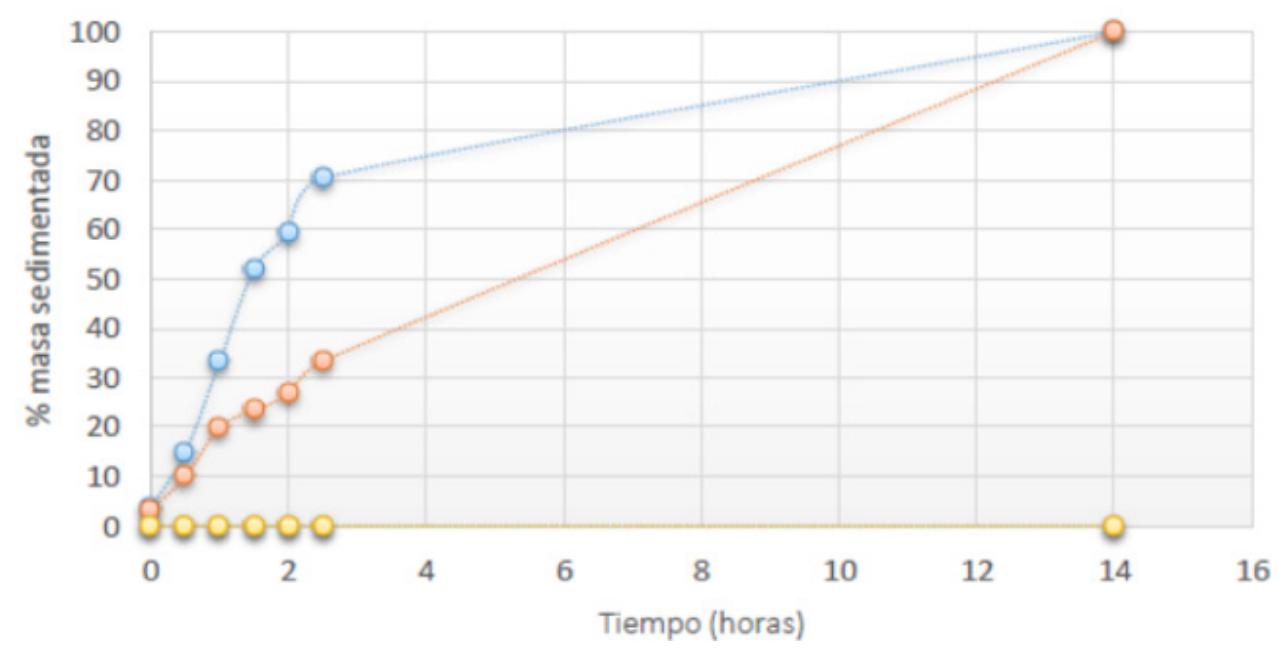

Fuente: Ensayos de Pre-tratamiento y tratamiento de efluentes en la empresa Pieles del Sur-Arequipa. 
- El ensayo del tratamiento físico químico se evaluó en función al nivel de DQO y de turbidez del efluente analizado; sin embargo, esta última variable no era un indicador adecuado por sí solo, ya que una determinada combinación de agentes podría generar un alto nivel de reducción de turbidez, mas no de DQO. La tabla 2 muestra los resultados de las 2 combinaciones con mejores resultados:

Tabla 2. DQO y turbidez en muestra de Flocpol003 + PAC y Flocpol003 + FeCL3

\begin{tabular}{|l|c|c|}
\hline \multicolumn{1}{|c|}{ Tratamiento } & DQO (mg/l) & Turbidez (NTU) \\
\hline Flocpol003 + PAC & 3.150 & 7 \\
\hline Flocpol003 + FeCL3 & 1.766 & 68 \\
\hline
\end{tabular}

Fuente: Ensayos de pretratamiento y tratamiento de efluentes en la empresa Pieles del Sur-Arequipa.

Los resultados del análisis de una muestra de efluentes en blanco arrojan un nivel de DQO de $5.133 \mathrm{mg} / \mathrm{l}$ y de turbidez de $542 \mathrm{NTU}$, evidenciando que, mediante el segundo tratamiento de la tabla 3 es posible lograr una reducción de aproximadamente el 65 \% de la concentración de DQO.

\subsection{Resultados del sistema de tratamiento}

Los resultados del último monitoreo de agua realizado sobre el sistema de tratamiento ya instalado y en operación se muestran en la tabla 3, en la que se comparan los valores de los parámetros a la entrada (punto de muestreo: cámara de ecualización) y a la salida (punto de muestreo: descarga del decantador) de la planta de tratamiento.

Tabla 3. Resultados de monitoreo de agua con el sistema implementado

\begin{tabular}{|l|l|c|c|c|}
\hline \multicolumn{1}{|c|}{ Ensayo } & \multicolumn{1}{c|}{ Unidad } & L.C.M & Entrada & Salida \\
\hline Aceites y grasas & $\mathrm{mg} / \mathrm{l}$ & 1.20 & 6.20 & 10.20 \\
\hline Cromo & $\mathrm{mg} / \mathrm{l}$ & 0.20 & 12.69 & 0.72 \\
\hline Cromo hexavalente & $\mathrm{mg} / \mathrm{l}$ & 0.010 & $<0.010$ & $<0.010$ \\
\hline Demanda bioquímica de oxígeno & $\mathrm{mg} / \mathrm{l}$ & 2.0 & 2253.0 & 910.5 \\
\hline Demanda química de oxígeno & $\mathrm{mg} / \mathrm{l}$ & 5 & 4377 & 1969 \\
\hline Nitrógeno amoniacal & $\mathrm{mg} \mathrm{N}-\mathrm{NH} / \mathrm{l}$ & 0.10 & 124.50 & 115.00 \\
\hline pH & Unidad de $\mathrm{pH}$ & 0.01 & 7.22 & 7.11 \\
\hline Sólidos suspendidos totales & $\mathrm{mg} / \mathrm{l}$ & 5 & 1660 & 174 \\
\hline Sulfuro & $\mathrm{mg} / \mathrm{l}$ & 0.020 & $<0.020$ & $<0.020$ \\
\hline Temperatura & ${ }^{\circ} \mathrm{C}$ & 0.01 & 18.7 & 16.6 \\
\hline
\end{tabular}

Fuente: Informe de Ensayo n.: IE-19-3730, Analytical Laboratory E.I.R.L.

Las mayores reducciones de se dieron en los niveles de cromo y de sólidos suspendidos totales, equivalentes al $94.3 \%$ y $89.5 \%$, respectivamente. El nivel de DBO se redujo en un 59.6 $\%$, mientras que el de DQO en un $55.0 \%$. Finalmente, el nitrógeno amoniacal y pH tuvieron reducciones menores equivalentes al $7.6 \%$ y $1.5 \%$; en el caso de los aceites y grasas se evidenció un incremento del $64.5 \%$, respecto del valor obtenido en la entrada. 


\section{Conclusiones y recomendaciones}

La realización de ensayos de oxidación de sulfuros, precipitación de cromo y prueba de jarras permitieron el diseño y evaluación del sistema antes de su implementación, seleccionando las operaciones e insumos que permitieron mejorar los resultados esperados respecto a la calidad de los efluentes.

Con la aplicación del sistema de tratamiento se alcanzaron altos niveles de reducción de diferentes contaminantes y agentes tóxicos, principalmente: cromo (94.3\%), sólidos suspendidos totales (89.5\%), DBO (59.6 \%) y DQO (55\%), demostrando que el tratamiento propuesto tiene un efecto altamente positivo en la calidad de los efluentes.

Finalmente, el tratamiento propuesto está basado en el tipo de tratamiento físico-químico, siendo recomendable evaluar la aplicación de un tratamiento biológico posterior que permitiría el cumplimiento de los parámetros exigidos por la normativa vigente aplicable.

\section{Referencias}

Abia, L., Rejo, M., y Vieira, R. (2003). La eliminación de sulfuros en el tratamiento de efluentes industriales peligrosos. Ingeniería Química - Dialnet (406): 2013-220.

Artiga, P. (2005). Contribución a la mejora del tratamiento biológico. Tesis de Doctorado. Universidad de Santiago de Compostela.

Córdova, H., Vargas, R., Cesare, F., Flores, L. y Visitación, L. (2014). Tratamiento de las aguas residuales del proceso de curtido tradicional y alternativo que utiliza acomplejantes de cromo. Revista De La Sociedad Química Del Perú, Scielo, vol. 80 (3): 183-191. Recuperado de http://www.scielo.org. pe/scielo.php?script=sci_arttext\&pid=\$1810-634X2014000300005

Haydar, S., y Anwar, J. (2009). Coagulation-flocculation studies of tannery wastewater using combination of alum with cationic and anionic polymers. Journal of Hazardous Materials-Science Direct, 168 (2-3): 1035-1040. Recuperado de https://www.sciencedirect.com/science/article/pii/ S0304389409003501?via\%3Dihub

Islam, N., Misbahuzzaman, K., Kamruzzaman, A. y Chakrabarty, M. (2011). Efficiency of different coagulants combination for the treatment of tannery effluents: A case study of Bangladesh. African Journal of Environmental Science and Technology-Researchgate, 5 (6): 409-419. Recuperado de https://www.researchgate.net/publication/267194179_Efficiency_of_different_coagulants_ combination_for_the_treatment_of_tannery_effluents___case_study_of_Bangladesh

Moral, A. (2010). Depuración de Cromo III en aguas residuales que contienen alta carga proteica. 10 Congreso Nacional Del Medio Ambiente CONAMA10-Researchgate, 1-10. Recuperado de https:// www.researchgate.net/publication/267335276_Depuracion_de_Cromo_III_en_aguas_residuales_ que_contienen_alta_carga_proteica

Ortiz, N. (2013). Recuperación y reutilización de cromo de las aguas residuales del proceso de curtido de curtiembres de San Benito (Bogotá), mediante un proceso sostenible y viable tecnológicamente Tesis de Maestría. Universidad de Manizales. Colombia.

Salas, G. (2005). Eliminación de sulfuros por oxidación en el tratamiento del agua residual de una curtiembre. Revista Peruana de Química e Ingeniería Química-UNMSM, 8 (1): 49-54. Recuperado de http://sisbib.unmsm.edu.pe/bibvirtual/publicaciones/ing_quimica/v08_n1/pdf/a08v8.pdf 